\title{
INTERSECTION BODIES AND THE BUSEMANN-PETTY PROBLEM
}

\author{
R. J. GARDNER
}

\begin{abstract}
It is proved that the answer to the Busemann-Petty problem concerning central sections of centrally symmetric convex bodies in $d$-dimensional Euclidean space $\mathbb{E}^{d}$ is negative for a given $d$ if and only if certain centrally symmetric convex bodies exist in $\mathbb{E}^{d}$ which are not intersection bodies. It is also shown that a cylinder in $\mathbb{E}^{d}$ is an intersection body if and only if $d \leq 4$, and that suitably smooth axis-convex bodies of revolution are intersection bodies when $d \leq 4$. These results show that the Busemann-Petty problem has a negative answer for $d \geq 5$ and a positive answer for $d=3$ and $d=4$ when the body witti smaller sections is a body of revolution.
\end{abstract}

\section{INTRODUCTION}

In recent years much attention has been paid to the class of centred zonoids, otherwise known as projection bodies. This subclass of the centrally symmetric convex bodies has surprising applications to several areas of mathematics, such as measure theory, combinatorics, functional analysis, and stochastic geometry, as well as to crystallography, stereology, and mathematical economics (see, for example, the articles [BL] by J. Bourgain and J. Lindenstrauss or [SW] by R. Schneider and W. Weil). In his article $\left[\mathrm{L}_{1}\right]$, E. Lutwak introduced the class of intersection bodies, which is in a sense dual to the class of projection bodies and for which it is natural to expect similarly wide applications. It is too early to say whether this expectation is fully justified, but the results of the present paper indicate that at the very least intersection bodies hold the key to one of the most intriguing unsolved problems of geometric tomography, the so-called Busemann-Petty problem.

In [BP], H. Busemann and C. M. Petty asked the following question, motivated by interest in area in Minkowskian geometry. Suppose $K_{1}$ and $K_{2}$ are convex bodies in $d$-dimensional Euclidean space $\mathbb{E}^{d}$ which are centrally symmetric with centre at the origin, and that

$$
\lambda_{d-1}\left(K_{1} \cap u^{\perp}\right) \leq \lambda_{d-1}\left(K_{2} \cap u^{\perp}\right),
$$

for all $u \in S^{d-1}$. Then is it true that

$$
\lambda_{d}\left(K_{1}\right) \leq \lambda_{d}\left(K_{2}\right) ?
$$

Received by the editors May 15, 1992.

1991 Mathematics Subject Classification. Primary 52A40, 53A65.

Key words and phrases. Convex body, section, Busemann-Petty problem, intersection body.

Research supported in part by NSF Grant DMS-9201508. 
(Here $\lambda_{i}$ denotes $i$-dimensional Lebesgue measure.) The Busemann-Petty problem has appeared in several places in the literature. See, for example, the articles $\left[\mathrm{B}_{2}\right],[\mathrm{K}],[\mathrm{MP}$, p. 99] and [Be, p. 663], and the books [BZ, p. 154] and [CFG, Problem A9, p. 22]. After a breakthrough by D. G. Larman and C. A. Rogers in [LR], and a series of fine papers by other authors, it is known that the answer is negative for $d \geq 7$, even if $K_{2}$ is a ball (see [Bo] by Bourgain or [G] by A. Giannopoulos; also [B, GR, and T]). On the other hand, there are some positive results. $\mathrm{H}$. Hadwiger and $\mathrm{M}$. Giertz independently showed, in $[\mathrm{H}]$ and [Gi] respectively, that the question has an affirmative answer when $K_{1}$ and $K_{2}$ are coaxial convex bodies of revolution in $\mathbb{E}^{3}$. Busemann and Petty noted in [BP] that the Busemann intersection inequality $\left.\left[\mathrm{B}_{1},(4), \mathrm{p} .2\right]\right)$ may be applied to obtain a positive answer to the Busemann-Petty problem when $K_{1}$ is an ellipsoid. Lutwak $\left[\mathrm{L}_{1}\right.$, Theorem 10.1] proved a far-reaching generalization of this fact by showing that the same is true whenever $K_{1}$ is an intersection body.

We prove here that the answer to the Busemann-Petty problem is negative for $d \geq 5$, and positive when $K_{1}$ is a body of revolution in $\mathbb{E}^{d}$ for $d=3$ or 4 . After obtaining these results, we received a preprint of the paper [P] by M. Papadimitrakis, in which counterexamples are also constructed for $d=5$ and $d=6$. His methods are in some ways similar to ours, in that they exploit the ability to invert integral transforms of rotationally symmetric functions. In other ways our approach is quite different, and we believe our formulation pinpoints the fundamental reason for the existence of such examples (see especially the final displayed inequality in the proof of Theorem 6.1). We are also able to exhibit the first completely explicit examples in less than seven dimensions, in Remark 6.4.

In our approach we focus on Lutwak's class of intersection bodies. We extend the results of Lutwak in $\left[\mathrm{L}_{1}\right]$ to prove that the answer to the Busemann-Petty problem is positive for a given $d$ if and only if every centrally symmetric convex body in $\mathbb{E}^{d}$, whose radial function is infinitely differentiable and whose Gaussian curvature is everywhere positive, is an intersection body (see Theorem 3.1). Intersection bodies are not required to be convex; in fact Lutwak's theorem mentioned above holds for star bodies, and this extension is crucial, as we show below (see Remark 5.2(ii)). In Theorem 6.1 we show that there is a quite simple geometric reason why a cylinder in $\mathbb{E}^{d}$ for $d \geq 5$ is not an intersection body. Counterexamples are then provided by approximating a cylinder by suitable convex bodies. To obtain our positive results, we show in Theorem 5.1 that under certain conditions, every centred star body of revolution in $\mathbb{E}^{d}$ for $d=3$ and 4 is an intersection body. Corollary 5.3 contains the results of $[\mathrm{H}]$ and [Gi], and without any restriction on the body $K_{2}$. Professor Papadimitrakis has informed the author that he also has some positive results in $\mathbb{E}^{d}$ for $d=3$ and 4 .

Since submission of this paper, there have been further developments. A new, and in some ways more appropriate, definition of the term intersection body has been proposed by Lutwak; see the next section. Moreover, the BusemannPetty problem is now settled in each dimension. For $d=4$, a negative answer has been obtained by Gaoyong Zhang (see $\left[Z_{2}, Z_{3}\right]$ ). Zhang achieves this by means of a characterization of intersection bodies (in the new sense) in terms of Lutwak's dual mixed volumes which he found earlier in $\left[Z_{1}\right]$. This character- 
ization allows him to conclude (see $\left[Z_{3}\right.$, Lemma 2$]$ ) that the differentiability and curvature assumptions of our Theorem 3.1 can actually be removed; thus, the Busemann-Petty problem has a positive answer in $\mathbb{E}^{d}$ if and only if each centrally symmetric convex body in $\mathbb{E}^{d}$ is an intersection body (in the new sense). Zhang then shows that in $\mathbb{E}^{d}, d \geq 4$, a cube is not an intersection body (in the new sense). Some of the other results of this paper are also re-proved by Zhang via his characterization of intersection bodies. For $d=3$, the present author proves in [Ga] that the answer to the Busemann-Petty problem is affirmative.

I thank Professors R. Schneider and V. Oliker for some helpful information concerning the differentiability properties of convex bodies.

\section{Preliminaries}

We denote the unit sphere and closed unit ball in $d$-dimensional Euclidean space $\mathbb{E}^{d}$ by $S^{d-1}$ and $B$, respectively. If $u \in S^{d-1}$, then $u^{\perp}$ is the subspace orthogonal to $u$. We write $\lambda_{i}$ for $i$-dimensional Lebesgue measure, which we identify with $i$-dimensional Hausdorff measure. We also let $\kappa_{d}=\lambda_{d}(B)$ and $\omega_{d}=\lambda_{d-1}\left(S^{d-1}\right)=d \kappa_{d}$.

As is usual, we denote by $C$ (or $C^{\infty}$ ) the class of continuous (or infinitely differentiable, respectively) functions. By $C_{e}$ or $C_{e}^{\infty}$ we mean the even functions in these classes.

Suppose $\phi$ is the vertical angle for spherical polar coordinates in $\mathbb{E}^{d}$; that is, the angle between a vector and the positive $x_{d}$-axis. We then say that a function $f$ on $S^{d-1}$ is rotationally symmetric (with respect to the $x_{d}$-axis) if its values depend only on $\phi$.

A convex body is a compact convex set with nonempty interior. A set $L$ is star-shaped at the origin if it contains the origin, and every line through the origin which meets $L$ does so in a (possibly degenerate) line segment. If $L$ is star-shaped at the origin, its radial function $\rho_{L}$ is defined by

$$
\rho_{L}(u)=\sup \{c \geq 0: c u \in L\},
$$

for $u \in S^{d-1}$. By a star body we mean a compact set which is star-shaped at the origin and whose radial function is continuous. We say a set is centred if it is centrally symmetric with centre at the origin. By a cylinder in $\mathbb{E}^{d}$ we shall always mean a right spherical cylinder; that is, the vector sum of a $(d-1)$ dimensional ball and an orthogonal closed line segment.

Suppose $L$ is a star body of revolution. Then $L$ is said to be axis-convex if each line parallel to its axis which meets it does so in a (possibly degenerate) line segment.

Suppose $g \in C\left(S^{d-1}\right)$, and $f$ is defined by

$$
f(u)=\int_{S^{d-1} \cap u^{\perp}} g(v) d \lambda_{d-2}(v),
$$

for all $u \in S^{d-1}$; that is, $f(u)$ is the integral of $g$ over the great sphere in $S^{d-1}$ orthogonal to $u$. Then we write

$$
f=R g
$$

and say that $f$ is the spherical Radon transform of $g$. The following useful fact is known about $R$ (see [He 1, p. 161]). 
2.1. Proposition. Suppose $f \in C_{e}^{\infty}\left(S^{d-1}\right)$. Then there is a $g \in C_{e}^{\infty}\left(S^{d-1}\right)$ such that $f=R g$.

It is also known (see $\left[\mathrm{He}_{1}\right.$, p. 144]) that $R$ is selfadjoint, in the sense that for $f$ and $g$ in $C_{e}\left(S^{d-1}\right)$,

$$
\int_{S^{d-1}} f(u)(R g)(u) d \lambda_{d-1}(u)=\int_{S^{d-1}}(R f)(u) g(u) d \lambda_{d-1}(u) .
$$

From this and the fact that by Proposition 2.1, the range of $R$ is dense in $C_{e}\left(S^{d-1}\right)$, it follows that $R$ is injective on $C_{e}\left(S^{d-1}\right)$. (Direct proofs of the injectivity are given in $\left[\mathrm{S}_{1}\right]$ and $[\mathrm{Pe}, \mathrm{p}$. 1546].) We shall also use the fact that $R$ commutes with rotations. This is proved in [Gr, p. 193] for the Radon transform on complex projective space, and the proof for $R$ is the same.

The star body $L$ is called an intersection body if there is another star body $M$ such that

$$
\rho_{L}(u)=\lambda_{d-1}\left(M \cap u^{\perp}\right),
$$

for all $u \in S^{d-1}$. We also say that $L$ is the intersection body of $M$ and write $L=I M$. It is known (see $\left[\mathrm{L}_{1},(8.3)\right]$ ) that in this case there is a unique such $M$ which is also centred. Clearly any intersection body is centred. It is also easy to see, using the polar coordinate formula for volume, that a star body $L$ is an intersection body if and only if $\rho_{L}=R g$ for some nonnegative continuous function $g$; just take $g=\rho_{M}^{d-1} /(d-1)$.

It is worth mentioning that Lutwak has offered as an alternative and different definition of intersection body, a star body $L$ such that $\rho_{L}=R \mu$, where $\mu$ is an even finite Borel measure in $S^{d-1}$. Though there are several reasons to prefer this definition, we shall adhere to the geometrically simpler definition in the previous paragraph.

The following theorem is Theorem 10.1 of $\left[\mathrm{L}_{1}\right]$.

2.2. Lutwak's theorem. Suppose $L_{1}$ is an intersection body and $L_{2}$ is an arbitrary star body, such that $\lambda_{d-1}\left(L_{1} \cap u^{\perp}\right) \leq \lambda_{d-1}\left(L_{2} \cap u^{\perp}\right)$, for all $u \in S^{d-1}$. Then

$$
\lambda_{d}\left(L_{1}\right) \leq \lambda_{d}\left(L_{2}\right) .
$$

\section{INTERSECTION BODIES AND THE BUSEMANN-PETTY PROBLEM}

3.1. Theorem. Let $d \geq 3$. The Busemann-Petty problem has a positive answer in $\mathbb{E}^{d}$ if and only if each centrally symmetric convex body $K$ in $\mathbb{E}^{d}$, with $\rho_{K} \in$ $C_{e}^{\infty}\left(S^{d-1}\right)$ and with everywhere positive Gaussian curvature, is an intersection body.

Proof. Suppose there are centred convex bodies $K_{1}$ and $K_{2}$ in $\mathbb{E}^{d}$ with $\lambda_{d-1}\left(K_{1} \cap u^{\perp}\right) \leq \lambda_{d-1}\left(K_{2} \cap u^{\perp}\right)$, for all $u \in S^{d-1}$, and $\lambda_{d}\left(K_{1}\right)>\lambda_{d}\left(K_{2}\right)$. By approximating, we can find a centrally symmetric convex body $K_{1}^{\prime}$, with $\rho_{K_{1}^{\prime}} \in C_{e}^{\infty}\left(S^{d-1}\right)$ and with everywhere positive Gaussian curvature, such that $K_{1}^{\prime} \subset K_{1}$ and $\lambda_{d}\left(K_{1}^{\prime}\right)>\lambda_{d}\left(K_{2}\right)$. To accomplish this approximation, we first appeal to a theorem of Schneider in $\left[S_{2}\right]$, which provides an approximating $K_{1}^{\prime}$ which is centred and has everywhere positive Gaussian curvature and an analytic boundary hypersurface. Now the map which takes $\rho_{K_{1}^{\prime}}(u) u \in \partial K_{1}^{\prime}$ to $u \in S^{d-1}$ is certainly $C^{\infty}$, and from this one can conclude that $\rho_{K_{1}^{\prime}} \in C_{e}^{\infty}\left(S^{d-1}\right)$. 
If $K_{1}^{\prime}$ is an intersection body, we must have $\lambda_{d-1}\left(K_{1}^{\prime} \cap u^{\perp}\right)>\lambda_{d-1}\left(K_{2} \cap u^{\perp}\right)$, for some $u \in S^{d-1}$, by Lutwak's theorem. This is impossible, so $K_{1}^{\prime}$ is not an intersection body.

Conversely, suppose there is a centrally symmetric convex body $K_{2}$ in $\mathbb{E}^{d}$, with $\rho_{K_{2}} \in C_{e}^{\infty}\left(S^{d-1}\right)$ and everywhere positive Gaussian curvature, which is not an intersection body. We follow the argument of Theorem 12.2 of $\left[\mathrm{L}_{1}\right]$. By Proposition 2.1, there is a $g \in C_{e}^{\infty}\left(S^{d-1}\right)$ such that $\rho_{K_{2}}=R g$. Choose an $F \in C_{e}^{\infty}\left(S^{d-1}\right)$ which is nonnegative when $g$ is negative, and zero otherwise. There is an $h \in C_{e}^{\infty}\left(S^{d-1}\right)$ such that $F=R h$, by Proposition 2.1 again. If $c>0$ is any constant such that $\rho_{K_{2}}^{d-1}-c h$ is positive on $S^{d-1}$, we define a star body $K_{1}$ (depending on $c$ ) by

$$
\rho_{K_{1}}^{d-1}=\rho_{K_{2}}^{d-1}-c h .
$$

In Theorem 12.2 of $\left[\mathrm{L}_{1}\right]$ it is shown that $\lambda_{d-1}\left(K_{1} \cap u^{\perp}\right) \leq \lambda_{d-1}\left(K_{2} \cap u^{\perp}\right)$, for all $u \in S^{d-1}$, but $\lambda_{d}\left(K_{1}\right)>\lambda_{d}\left(K_{2}\right)$. Therefore it only remains to prove that for suitable $c$ the star body $K_{1}$ is actually convex. By the definition of $\rho_{K_{1}}$, it and any of its derivatives converge uniformly to $\rho_{K_{2}}$ and its corresponding derivatives, respectively, as $c$ tends to zero. In [O, 2.5] one can find a formula for the Gaussian curvature of a star body containing the origin in terms of its radial function, the numerator of which is a determinant whose entries involve only the radial function and its first and second derivatives. Consequently, since the Gaussian curvature of $K_{2}$ is positive everywhere, we can find a $c$ which is small enough to ensure that that of $K_{1}$ is also positive everywhere. It then follows from a standard result (see, for example, [KN, p. 41]) that $K_{1}$ is convex.

\section{AN INVERSION FORMULA FOR THE SPHERICAL RADON TRANSFORM}

Suppose that $f \in C_{e}\left(S^{d-1}\right)$ is rotationally symmetric with respect to the $x_{d}$-axis, and $f=R g$ for $g \in C_{e}\left(S^{d-1}\right)$. Then, using the facts (see §2) that $R$ is injective on $C_{e}\left(S^{d-1}\right)$ and commutes with rotations, we see that $g$ is also rotationally symmetric with respect to the $x_{d}$-axis. In this section we outline the known procedure for finding $g$ in terms of $f$.

Let us fix a $u \in S^{d-1}$. Let $p$ be a point in the $(d-2)$-sphere $u^{\perp} \cap S^{d-1}$, such that the line $l$ through $p$ and the origin has a minimal angle $\psi$ with the $x_{d}$-axis. Then the angle between $u$ and the $x_{d}$-axis is $\pi / 2-\psi$. Denote by $\alpha$ the vertical angle for spherical polar coordinates in $u^{\perp}$, where we take the line $l$ as the vertical axis positively oriented in the direction of $p$; that is, $\alpha$ denotes the angle between a vector in $u^{\perp}$ and the ray from the origin through $p$. Let $q$ be any vector in $u^{\perp} \cap S^{d-1}$, and suppose $\phi$ and $\alpha$ are its angles with the $x_{d}$-axis and $l$, respectively. Then

$$
\cos \phi=\cos \alpha \cos \psi \text {. }
$$

Now the function $g$ has the same value at any vector in $u^{\perp} \cap S^{d-1}$ which has the same coordinate $\alpha$. The set of all such vectors forms a $(d-3)$-sphere of radius $\sin \alpha$. Using the evenness of $g$, the equation $f=R g$ then becomes

$$
f\left(\frac{\pi}{2}-\psi\right)=2 \omega_{d-2} \int_{0}^{\pi / 2} g(\phi) \sin ^{d-3} \alpha d \alpha,
$$


for $0 \leq \psi<\pi / 2$, and we have $f(0)=\omega_{d-1} g(\pi / 2)$. We substitute $x=\cos \psi$ and $t=\cos \phi$ in the integral to obtain

$$
f\left(\sin ^{-1} x\right)=\frac{2 \omega_{d-2}}{x^{d-3}} \int_{0}^{x} g\left(\cos ^{-1} t\right)\left(x^{2}-t^{2}\right)^{(d-4) / 2} d t
$$

for $0<x \leq 1$, and $f\left(\sin ^{-1} 0\right)=\omega_{d-1} g\left(\cos ^{-1} 0\right)$. With differences only in notation, the last displayed equation is $(3.10)$ of $\left[\mathrm{He}_{2}\right]$. The equation is inverted by standard techniques, assuming the appropriate order of differentiability for $f$, to give the following formula (cf. $\left[\mathrm{He}_{2},(3.11)\right]$ ).

\subsection{Inversion formula.}

$$
g\left(\cos ^{-1} t\right)=\frac{1}{(d-3) ! \omega_{d-1}} t\left(\frac{1}{t} \frac{d}{d t}\right)^{d-2} \int_{0}^{t} f\left(\sin ^{-1} x\right) x^{d-2}\left(t^{2}-x^{2}\right)^{(d-4) / 2} d x
$$

for $0<t \leq 1$, and $g\left(\cos ^{-1} 0\right)=f\left(\sin ^{-1} 0\right) / \omega_{d-1}$.

\section{Positive Results}

5.1. Theorem. Let $d=3$ or 4 , and suppose that $L$ is an axis-convex centred star body of revolution in $\mathbb{E}^{d}$ with $\rho_{L} \in C_{e}^{\infty}\left(S^{d-1}\right)$. Then $L$ is an intersection body.

Proof. We may suppose that $L$ is a body of revolution about the $x_{d}$-axis. By Proposition 2.1, there is a $g \in C_{e}^{\infty}\left(S^{d-1}\right)$ with $\rho_{L}=R g$.

Let us first consider $d=3$. The inversion formula states that

$$
g\left(\cos ^{-1} t\right)=\frac{1}{2 \pi} \frac{d}{d t} \int_{0}^{t} \frac{x \rho_{L}\left(\sin ^{-1} x\right)}{\sqrt{t^{2}-x^{2}}} d x,
$$

for $0<t \leq 1$, and $g\left(\cos ^{-1} 0\right)=\rho_{L}\left(\sin ^{-1} 0\right) / 2 \pi$. We have to show that $g$ is nonnegative, and this will follow if the integral increases with $t$. Under the substitution $s=x / t$, the integral becomes

$$
\int_{0}^{1} \frac{s t \rho_{L}\left(\sin ^{-1}(s t)\right)}{\sqrt{1-s^{2}}} d s
$$

But the axis-convexity of $L$ means that $\sin \phi \rho_{L}(\phi)$ increases with $\phi$, so $x \rho_{L}\left(\sin ^{-1} x\right)$ increases with $x$, and the result follows.

Now suppose that $d=4$. Rather than apply the inversion formula directly, it is easier in this case to note that (1) above, with $f=\rho_{L}$, becomes

$$
\rho_{L}\left(\sin ^{-1} x\right)=\frac{4 \pi}{x} \int_{0}^{x} g\left(\cos ^{-1} t\right) d t
$$

for $0<x \leq 1$, and $\rho_{L}\left(\sin ^{-1} 0\right)=4 \pi g\left(\cos ^{-1} 0\right)$. Multiplying by $x$ and differentiating with respect to $x$, we obtain

$$
g\left(\cos ^{-1} x\right)=\frac{1}{4 \pi} \frac{d}{d x}\left(x \rho_{L}\left(\sin ^{-1} x\right)\right),
$$

for $0<x \leq 1$, and $g\left(\cos ^{-1} 0\right)=\rho_{L}\left(\sin ^{-1} 0\right) / 4 \pi$. The axis-convexity of $L$ immediately implies that $g$ is nonnegative, as required. 
5.2. Remark. (i) We take the trouble to state the previous theorem with the axis-convexity condition since similar conditions are used in both [H] and [Gi]. Some such condition is certainly necessary, since the theorem is false for star bodies satisfying the remaining conditions. This is not difficult to see from $[\mathrm{H}$, Example 3(a)] and Lutwak's theorem.

(ii) It is important to note that even when $K$ is a convex intersection body, the unique centred star body $M$ for which $K=I M$ need not be convex. A specific example of this phenomenon is provided by the cylinder $K$ in $\mathbb{E}^{3}$ obtained by rotating the centred square of side 2 in the $\left(x_{1}, x_{3}\right)$-plane about the $x_{3}$-axis. Then $M$ can be computed explicitly from the inversion formula 4.1 (which, it should be clear, only requires continuity of $f$ for $d=3$ ). We find that $M$ is the star body of revolution about the $x_{3}$-axis whose radial function $\rho_{M}(\phi)$, where $\phi$ is the vertical angle from the positive $x_{3}$-axis, is

$$
\rho_{M}(\phi)= \begin{cases}\frac{1}{\sqrt{\pi}}\left(\frac{1}{1-\cos \phi}+\frac{\sin ^{2} \phi \sec \phi}{\sqrt{\cos 2 \phi}-\cos ^{2} \phi}\right)^{1 / 2} & \text { if } 0<\phi \leq \frac{\pi}{4}, \\ \frac{\csc \phi}{\sqrt{\pi}} & \text { if } \frac{\pi}{4} \leq \phi \leq \frac{\pi}{2},\end{cases}
$$

and $\rho_{M}(0)=1 / \sqrt{2 \pi}$. Therefore $M$ is a centred cylinder of radius $1 / \sqrt{\pi}$ and height $2 / \sqrt{\pi}$, with its flat top and bottom replaced by certain surfaces, concave towards its centre-a "dented tin can" which has the points $(0,0, \pm 1 / \sqrt{2 \pi})$ in its boundary. See also the four-dimensional case in Remark 6.2.

5.3. Corollary. Let $d=3$ or 4 , and suppose that $L_{1}$ is an axis-convex centred star body of revolution in $\mathbb{E}^{d}$. If $L_{2}$ is an arbitrary star body, and

$$
\lambda_{d-1}\left(L_{1} \cap u^{\perp}\right) \leq \lambda_{d-1}\left(L_{2} \cap u^{\perp}\right), \quad \text { for all } u \in S^{d-1},
$$

then $\lambda_{d}\left(L_{1}\right) \leq \lambda_{d}\left(L_{2}\right)$.

Proof. As in the proof of Theorem 3.1, we may, by approximating if necessary, assume that $\rho_{L_{1}} \in C_{e}^{\infty}\left(S^{d-1}\right)$. (Suppose the $x_{d}$-axis is the axis of $L_{1}$, and let $M_{1}$ be the intersection of $L_{1}$ and the $\left\{x_{1}, x_{d}\right\}$-plane. The boundary of $M_{1}$ is given by two continuous functions of $x_{1}$, so by standard techniques one can find an axis-convex centred star body $M_{1}^{\prime}$, with $M_{1}^{\prime} \subset M_{1}$, such that $M_{1}^{\prime}$ is symmetric about the $x_{d}$-axis and $\rho_{M_{1}^{\prime}} \in C_{e}^{\infty}\left(S^{1}\right)$. The body $L_{1}^{\prime}$ obtained by rotating $M_{1}^{\prime}$ about the $x_{d}$-axis is then the required approximation.) By Theorem 5.1, $L_{1}$ is an intersection body, and the corollary follows from Lutwak's theorem.

\section{NegAtive RESUlts}

6.1. Theorem. If $d \geq 5$, a cylinder in $\mathbb{E}^{d}$ is not an intersection body.

Proof. Let $K$ be a cylinder in $\mathbb{E}^{d}, d \geq 5$. Every intersection body is centred, and the class of intersection bodies is preserved under linear transformations (see $\left[\mathrm{L}_{2}\right.$, p. 22]). We may therefore suppose that $K$ is the Cartesian product of the centred unit $(d-1)$-dimensional ball in the plane $x_{d}=0$ and the line segment $[-1,1]$ in the $x_{d}$-axis.

Suppose that $K$ is an intersection body. Then there is a unique centred star body $M$ such that $K=I M$. This is equivalent to $f=R g$, where $f=\rho_{K}$ 
and $g=\rho_{M}^{d-1} /(d-1)$. Since $R$ commutes with rotations, $M$ is also a body of revolution about the $x_{d}$-axis. Now $\rho_{K}(0)=1$, so $\rho_{M}(\pi / 2)=\kappa_{d-1}^{-1 /(d-1)}$. Furthermore, $\rho_{K}(\phi)=\sec \phi$ for $0 \leq \phi \leq \pi / 4$. This implies that $\rho_{M}(\phi)=$ $\kappa_{d-1}^{-1 /(d-1)} \csc \phi$ for $\pi / 4 \leq \phi \leq \pi / 2$, since the corresponding central sections of $M$ then have the correct $\lambda_{d-1}$-measure.

Geometrically, this means that $M$ must contain the body $M^{\prime}$ obtained by rotating about the $x_{d}$-axis the triangle in the $\left(x_{1}, x_{d}\right)$-plane with vertices at the origin and $\left(\kappa_{d-1}^{-1 /(d-1)}, \pm \kappa_{d-1}^{-1 /(d-1)}\right)$. Let us compute $\lambda_{d-1}\left(M^{\prime} \cap x_{1}^{\perp}\right)$, where $x_{1}^{\perp}$ is the hyperplane orthogonal to the $x_{1}$-axis. This is a $(d-1)$-dimensional cylinder with two $(d-1)$-dimensional cones removed, so we easily find that

$$
\begin{aligned}
\lambda_{d-1}\left(M^{\prime} \cap x_{1}^{\perp}\right) & =2\left(\kappa_{d-1}^{-1 /(d-1)}\right)^{d-1} \kappa_{d-2}-\frac{2}{d-1}\left(\kappa_{d-1}^{-1 /(d-1)}\right)^{d-1} \kappa_{d-2} \\
& =\frac{2(d-2) \kappa_{d-2}}{(d-1) \kappa_{d-1}} .
\end{aligned}
$$

Now

$$
\lambda_{d-1}\left(M^{\prime} \cap x_{1}^{\perp}\right) \leq \lambda_{d-1}\left(M \cap x_{1}^{\perp}\right)=\rho_{K}(\pi / 2)=1,
$$

so we must have

$$
a_{d}=\frac{2(d-2) \kappa_{d-2}}{(d-1) \kappa_{d-1}} \leq 1 .
$$

However, it is easily shown that $a_{d+2} \geq a_{d}$, for all $d \geq 1$ and one can calculate that $a_{5}=4 / \pi>1$ and $a_{6}=3 / 2>1$.

6.2. Remark. It is interesting to apply the argument of Theorem 6.1 when $d=4$. In this case we find that

$$
2(d-2) \kappa_{d-2} /\left((d-1) \kappa_{d-1}\right)=4 \kappa_{2} / 3 \kappa_{3}=1 \text {. }
$$

The geometry behind this is clear; the volume of a three-dimensional circular cylinder of equal height and radius, less that of a right cone of the same height and base, equals the volume contained in a hemisphere of the same radius as the cylinder, as Archimedes knew well. So $M=M^{\prime}$, and $M$ is the star-shaped (at the origin) body of revolution about the $x_{4}$-axis obtained by rotating the triangle in the $\left(x_{1}, x_{4}\right)$-plane with vertices at the origin and $\left(\kappa_{3}^{-1 / 3}, \pm \kappa_{3}^{-1 / 3}\right)$. (Note that $M$ is technically not a star body by our definition, since it does not have a continuous radial function.) This example also shows that the Busemann-Petty problem only just has a positive answer when $K_{1}$ is a body of revolution and $d=4$.

6.3. Theorem. If $d \geq 5$, the Busemann-Petty problem has a negative answer.

Proof. Let $d \geq 5$. By Theorem 3.1, it is enough construct a centrally symmetric convex body in $\mathbb{E}^{d}$ which is not an intersection body and which in addition has a $C^{\infty}$ radial function and everywhere positive Gaussian curvature. The cylinder $K$ from Theorem 6.1 fulfills these conditions, except for the last two. Let us therefore consider any sequence $\left(K_{n}\right)$ of centred convex bodies of revolution about the $x_{d}$-axis which satisfies the following properties:

(i) each $K_{n}$ has $\rho_{K_{n}} \in C^{\infty}\left(S^{d-1}\right)$ and everywhere positive Gaussian curvature;

(ii) the bodies $K_{n}$ converge to $K$ in the Hausdorff metric; 
(iii) for any $\varepsilon>0$, the functions $\rho_{K_{n}}(\phi)$ and all their derivatives converge uniformly to $\rho_{K}(\phi)$ and all its corresponding derivatives, respectively, as $n$ tends to infinity, for $0 \leq \phi \leq \pi / 4-\varepsilon$ (and therefore also for $3 \pi / 4+\varepsilon \leq \phi \leq \pi$ ). Here, as before, $\phi$ denotes the angle from the $x_{d}$-axis.

Property (iii) can be obtained by taking the appropriate parts of the boundary of $K_{n}$ to be caps of spheres centred on the $x_{d}$-axis and with radii approaching infinity with $n$, for example. The condition ensures, in view of the inversion formula 4.1, that if $\rho_{K_{n}}=R g_{n}$, then for each $\varepsilon>0, g_{n}(\phi)$ converges uniformly to $g(\phi)$ for $\pi / 4+\varepsilon \leq \phi \leq 3 \pi / 4-\varepsilon$, as $n$ tends to infinity; here $g$ is as in Theorem 6.1.

Let $0<\delta<4 / \pi-1$. It is now a straightforward matter to find a sufficiently large $n$ so that when the proof of Theorem 6.1 is applied to $K_{n}$ instead of $K$ we conclude that

$$
2(d-2) \kappa_{d-2} /\left((d-1) \kappa_{d-1}\right) \leq 1+\delta<4 / \pi .
$$

This contradiction completes the proof.

6.4. Remark. As in [P], our proof above does not provide explicit counterexamples. We can however prove that when $d=6$ one can take $K_{1}$ to be the cylinder from Theorem 6.1 and $K_{2}$ to be the convex body of revolution about the $i_{6}$-axis obtained by rotating the curve in the $\left(x_{1}, x_{6}\right)$-plane whose equation is

where

$$
\left|x_{1}\right|^{n}+\left|x_{6}\right|^{n}=a^{n}
$$

$$
a=\left(\frac{n \Gamma(1+6 / n)}{\Gamma(1+5 / n) \Gamma(1 / n)}\right)^{1 / 6}
$$

and $n \geq 13$. The idea is to follow the method used by A. Giannopoulos in [G] for a cylinder and ball in $\mathbb{E}^{d}$ with $d \geq 7$. The constant $a$ is chosen so that the volumes of $K_{1}$ and $K_{2}$ are equal, and one then shows that $K_{1}$ has strictly smaller sections. (One would then strictly speaking have to shrink $K_{2}$ slightly to obtain counterexamples to the problem as it is formulated in the introduction.) The details are too lengthy to be given here. For $d=5$, it appears possible to use the same method, but here our proof is not complete, since a certain difference of integrals is less amenable to analysis than in the case $d=6$. Numerical evidence suggests, however, that when $d=5$ one can again take $K_{1}$ to be the cylinder from Theorem 6.1 and $K_{2}$ to be the convex body of revolution about the $x_{5}$-axis obtained by rotating the curve in the $\left(x_{1}, x_{5}\right)$-plane whose equation is

where

$$
\left|x_{1}\right|^{n}+\left|x_{5}\right|^{n}=b^{n}
$$

$$
b=\left(\frac{n \Gamma(1+5 / n)}{\Gamma(1+4 / n) \Gamma(1 / n)}\right)^{1 / 5}
$$

and $n \geq i$.

\section{REFERENCES}

[B] K. Ball, Some remarks on the geometry of convex sets, Geometric Aspects of Functional Analysis, (J. Lindenstrauss and V. D. Milman, eds.), Lecture Notes in Math., vol. 1317, Springer-Verlag, Berlin and New York, 1988, pp. 224-231.

[Be] M. Berger, Convexity, Amer. Math. Monthly 97 (1990), 650-678. 
[Bo] J. Bourgain, On the Busemann-Petty problem for perturbations of the ball, Geom. Funct. Anal. 1 (1991), 1-13.

[BL] J. Bourgain and J. Lindenstrauss, Projection bodies, Geometric Aspects of Functional Analysis, (J. Lindenstrauss and V. D. Milman, eds.), Lecture Notes in Math., vol. 1317, SpringerVerlag, Berlin and New York, 1988, pp. 250-270.

[BZ] Yu. D. Burago and V. A. Zalgaller, Geometric inequalities, Springer-Verlag, Berlin and New York, 1988.

$\left[\mathrm{B}_{1}\right]$ H. Busemann, Volume in terms of concurrent cross-sections, Pacific J. Math. 3 (1953), 1-12.

[B $\left.\mathrm{B}_{2}\right]$, Volumes and areas of cross-sections, Amer. Math. Monthly 67 (1960), 248-250; correction 67 (1960), 671.

[BP] H. Busemann and C. M. Petty, Problems on convex bodies, Math. Scand. 4 (1956), 88-94.

[CFG] H. T. Croft, K. J. Falconer, and R. K. Guy, Unsolved problems in geometry, Springer-Verlag, Berlin and New York, 1991.

[F] P. Funk, Über Flächen mit lauter geschlossenen geodätischen Linien, Math. Ann. 74 (1913), 278-300.

[Ga] R. J. Gardner, A positive answer to the Busemann-Petty problem in three dimensions (to appear).

[G] A. A. Giannopoulos, A note on a problem of H. Busemann and C. M. Petty concerning sections of symmetric convex bodies, Mathematika 37 (1990), 239-244.

[Gi] M. Giertz, A note on a problem of Busemann, Math. Scand. 25 (1969), 145-148.

[GLW] P. R. Goodey, E. Lutwak and W. Weil, Functional analytic characterizations of classes of convex bodies (to appear).

[Gr] E. L. Grinberg, Spherical harmonics and integral geometry on projective spaces, Trans. Amer. Math. Soc. 279 (1983), 187-203.

[GR] E. L. Grinberg and I. Rivin, Infinitesimal aspects of the Busemann-Petty problem, Bull. London Math. Soc. 22 (1990), 478-484.

[H] H. Hadwiger, Radialpotenzintegrale zentralsymmetrischer Rotationskörper und ungleichheitaussagen Busemannischer Art, Math. Scand. 23 (1968), 193-200.

[He $\left.{ }_{1}\right]$ S. Helgason, Groups and geometric analysis, Academic Press, San Diego, 1984.

$\left[\mathrm{He}_{2}\right] \ldots$, The totally-geodesic Radon transform on constant curvature spaces, Contemp. Math. 113 (1990), 141-149.

[K] V. L. Klee, Ungelöstes Problem Nr. 44, Elem. Math. 17 (1962), 84.

[KN] S. Kobayashi and K. Nomizu, Foundations of differential geometry, vol. 2, Wiley, New York, 1969.

[LR] D. G. Larman and C. A. Rogers, The existence of a centrally symmetric convex body with central sections that are unexpectedly small, Mathematika 22 (1975), 164-175.

$\left[\mathrm{L}_{1}\right] \quad$ E. Lutwak, Intersection bodies and dual mixed volumes, Adv. Math. 71 (1988), 232-261.

[ $\left.\mathrm{L}_{2}\right]$, On some ellipsoid formulas of Busemann, Furstenberg and Tzkoni, Guggenheimer, and Petty, J. Math. Anal. Appl. 159 (1991), 18-26.

[MP] V. D. Milman and A. Pajor, Isotropic position and inertia ellipsoids and zonoids of the unit ball of a normed n-dimensional space, Geometric Aspects of Functional Analysis, (J. Lindenstrauss and V. D. Milman, eds.), Lecture Notes in Math., vol. 1376, Springer-Verlag, Berlin and New York, 1989, pp. 64-104.

[O] V. I. Oliker, Hypersurfaces in $\mathbb{R}^{n+1}$ with prescribed Gaussian curvature and related equations of Monge-Ampere type, Comm. Partial Differential Equations 9 (1984), 807-838.

[P] M. Papadimitrakis, On the Busemann-Petty problem about convex, centrally symmetric bodies in $\mathbb{R}^{n}$, Mathematika (to appear).

[Pe] C. M. Petty, Centroid surfaces, Pacific J. Math. 11 (1961), 1535-1547.

$\left[\mathrm{S}_{1}\right] \quad \mathrm{R}$. Schneider, Functions on a sphere with vanishing integrals over certain subspheres, J. Math. Anal. Appl. 26 (1969), 381-384.

[ $\left.\mathrm{S}_{2}\right] \longrightarrow$ Smooth approximations of convex bodies, Rend. Circ. Mat. Palermo (2) 33 (1984), 436-440. 
[SW] R. Schneider and W. Weil, Convexity and Its Applications, (P. M. Gruber and J. M. Wills, eds.), Birkhäuser, Basel, 1983, pp. 296-317.

[T] S. Tanno, Central sections of centrally symmetric convex bodies, Kodai Math. J. 10 (1987), 343-361.

$\left[Z_{1}\right]$ Gaoyong $Z$ hang, Centered bodies and dual mixed volumes (to appear).

$\left[\mathrm{Z}_{2}\right] \quad$, Intersection bodies and the four-dimensional Busemann-Petty problem, Duke Math. J. 71 (1993), 233-240.

$\left[Z_{3}\right] \quad$, Intersection bodies and the Busemann-Petty inequalities in $\mathbb{R}^{4}$, Ann. of Math. (to appear).

Department of Mathematics, Western Washington University, Bellingham, WashingTON 98225-9063

E-mail address: gardner@baker.math. wru. edu 